\title{
Karyotype variations in Italian populations of the peach-potato aphid Myzus persicae (Hemiptera: Aphididae)
}

\author{
M. Rivi ${ }^{1}$, V. Monti ${ }^{1,2}$, E. Mazzoni ${ }^{3}$, S. Cassanelli ${ }^{1}$, \\ M. Panini ${ }^{3}$, D. Bizzaro ${ }^{4}$, M. Mandrioli ${ }^{2}$ \\ and G.C. Manicardi ${ }^{1 *}$ \\ ${ }^{1}$ Dipartimento di Scienze Agrarie e degli Alimenti, Università di Modena e \\ Reggio Emilia, Reggio Emilia, Italy: ${ }^{2}$ Dipartimento di Biologia, Università di \\ Modena e Reggio Emilia, Modena, Italy: ${ }^{3}$ Istituto di Entomologia e Patologia \\ vegetale, Università Cattolica del Sacro Cuore, Piacenza, Italy: ${ }^{4}$ Dipartimento \\ di Scienze della Vita e dell'Ambiente, Università Politecnica delle Marche, \\ Ancona, Italy
}

\begin{abstract}
In this study, we present cytogenetic data regarding 66 Myzus persicae strains collected in different regions of Italy. Together with the most common $2 n=12$ karyotype, the results showed different chromosomal rearrangements: $2 n=12$ with A1-3 reciprocal translocation, $2 n=13$ with A1-3 reciprocal translocation and A3 fission, $2 n=13$ with A3 fission, $2 n=13$ with A4 fission, $2 n=14$ with $\mathrm{X}$ and A3 fissions. A 2n=12-13 chromosomal mosaicism has also been observed. Chromosomal aberrations (and in particular all strains showing A1-3 reciprocal translocation) are especially frequent in strains collected on tobacco plants, and we suggest that a clastogenic effect of nicotine, further benefited by the holocentric nature of aphid chromosomes, could be at the basis of the observed phenomenon.
\end{abstract}

Keywords: karyotype variations, chromosomal rearrangements, holocentric chromosomes, nicotine, clastogenic effect, Myzus persicae, Aphididae

(Accepted 27 March 2012; First published online 30 May 2012)

\section{Introduction}

Classical and molecular cytogenetics provide an integrated approach for structural, functional and evolutionary analyses of chromosomes. This ranges from karyotype analyses to molecular mapping of chromosomes.

To date, studies concerning chromatin structure and organization have been mainly focused on eukaryotes having monocentric chromosomes, whereas species possessing holocentric/holokinetic chromosomes have been rather

*Author for correspondence

Fax: +39-0522-522027

E-mail: giancarlo.manicardi@unimore.it neglected. Chromosomes with diffused centromeric activity have been found in Protista, as well as in plant and animal species (Wrensch et al., 1994). The chromosomes of aphids, like those of other hemipteran insects, have diffuse centromeres so that kinetic activity is dispersed along the entire length of each chromatid at least in mitotic divisions, thus influencing chromosome behaviour (White, 1973). In organisms possessing this kind of chromatin organization, chromosome fusions and fissions can occur without any duplication or loss of centromeres. This has consequences for the survival of the de novo chromosomal changes through mitosis and meiosis, and hence for karyotype evolution. Autosomal fusions and fissions, particularly the latter, seemed to play a pivotal role in aphid karyotype evolution (Blackman, 1980), although this view is at present somewhat speculative due 
Table 1. List of the Italian populations of M. persicae analyzed.

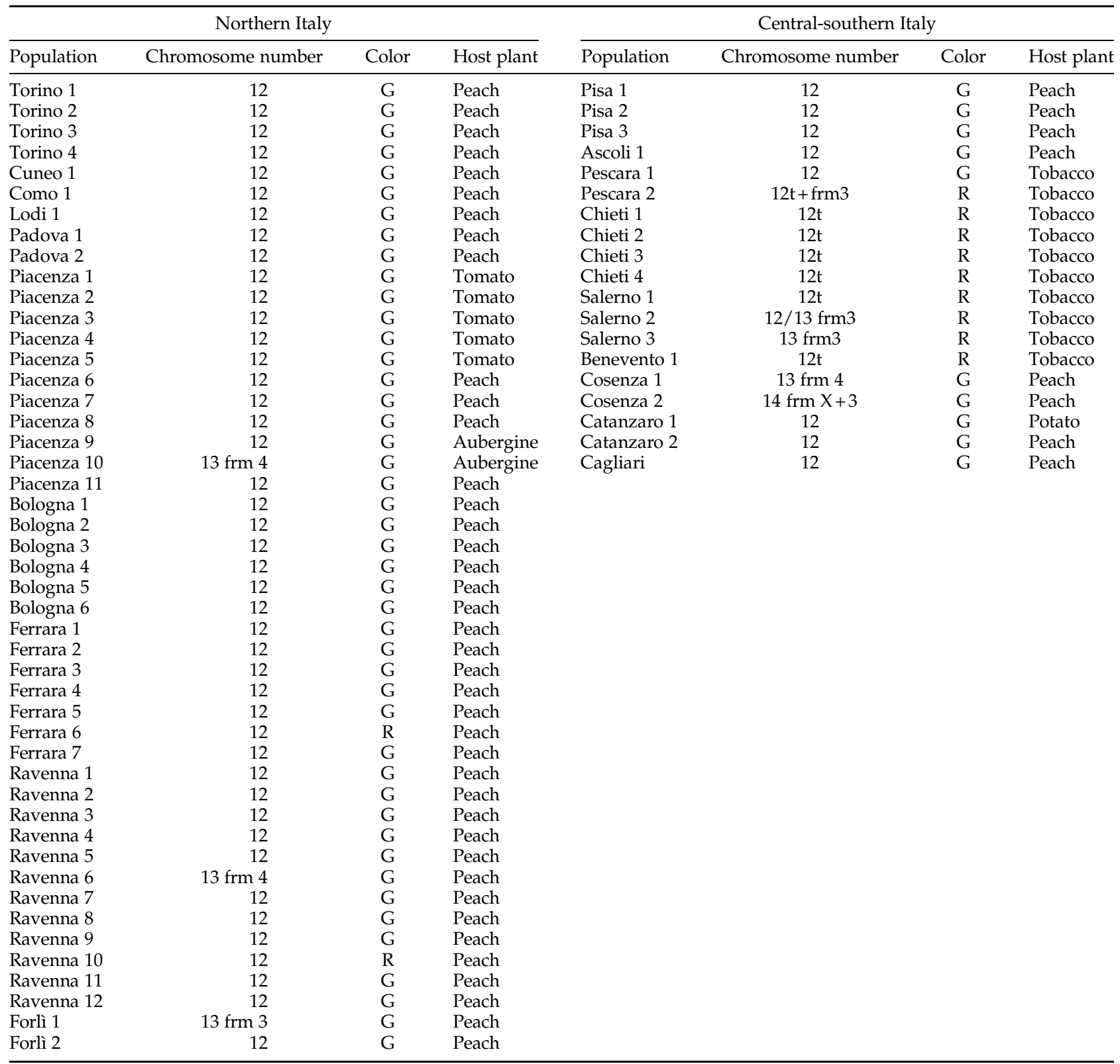

to a lack of knowledge concerning the mechanisms involved in rearrangements of the holocentric chromosomes (Spence \& Blackman, 2000).

A recurrent chromosomal rearrangement found in the peach-potato aphid Myzus persicae (Sulzer) (Hemiptera: Aphididae) populations collected worldwide involves a A1-3 reciprocal translocation associated with increased levels of resistance to organophosphate and carbamate insecticides (Blackman et al., 1978; Spence \& Blackman, 1998).

The standard female karyotype of this species is $2 n=12$, but specimens with a chromosome complement of either $2 n=13$ or 14 have also been reported (Blackman, 1980; Lauritzen, 1982). On the basis of relative chromosome lengths, Blackman (1971) concluded that the $2 n=13$ karyotype raised from a break in one autosome of the pair A3, whereas a break in one chromosome of either the A2 and A3 pairs led to a $2 n=14$ karyotype. Rare cases of strain possessing $2 n=11$ and $3 n=18$ have also been reported (Blackman, 1980; Yang \& Zhang, 2000). Very recently, the analysis of mitotic metaphase chromosomes of a $M$. persicae laboratory strain revealed different chromosome numbers, ranging from 12 to 17, within each embryo (intraclonal genetic variation sensu Loxdale \& Lushai (2003)). Chromosome length measurements revealed that the observed chromosomal mosaicism is due to recurrent fragmentations of chromosomes X, 1 and 3 (Monti et al., 2012).

The present study shows cytogenetic data regarding 66 $M$. persicae strains collected in different Italian regions showing several chromosomal rearrangements, the most 


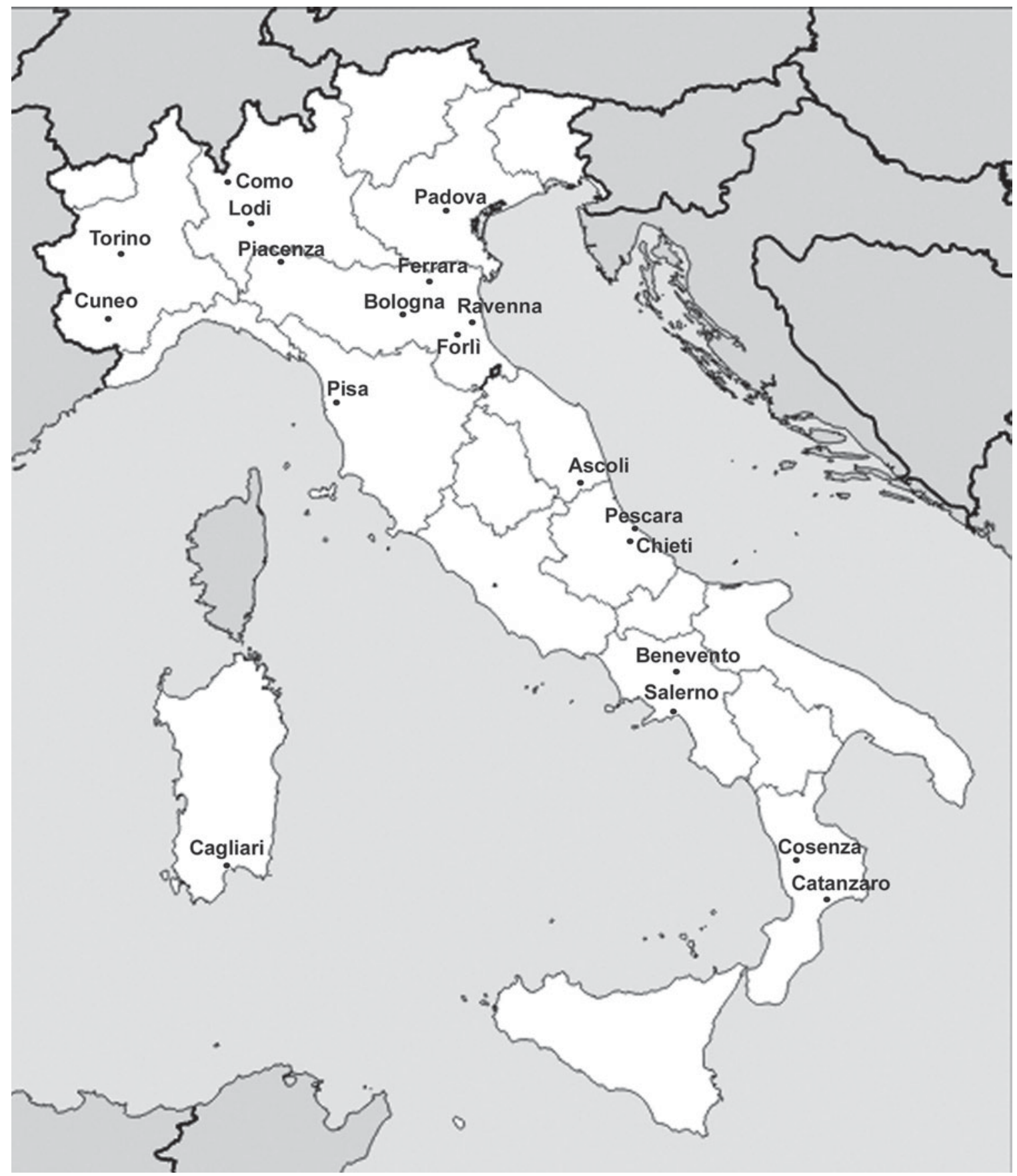

Fig. 1. Geographic distribution of the sampling sites.

common being the A1-3 reciprocal translocation, which we here reported for the first time in Italy. We have also looked for the presence of a relationship between karyotype variations and the host plants.

\section{Material and methods}

Myzus persicae populations were collected mainly from peach (Prunus persica (L.) Batsch) orchards (48), but also from herbaceous hosts like tobacco (10), tomato (5), potato (1) and aubergine (2) at various locations in different areas of Italy (see table 1, fig. 1) and maintained as parthenogenetic female colonies on pea-seedlings (Pisum sativum cv 'Meraviglia d'Italia') under constant environmental conditions: $21^{\circ} \mathrm{C}$, $16 \mathrm{~h}$ light:8 h dark photoperiod.

For chromosome spreads, adult females were dissected in Ringer saline solution and embryos were kept in a $1 \%$ hypotonic solution of sodium citrate for $30 \mathrm{~min}$. The embryos 


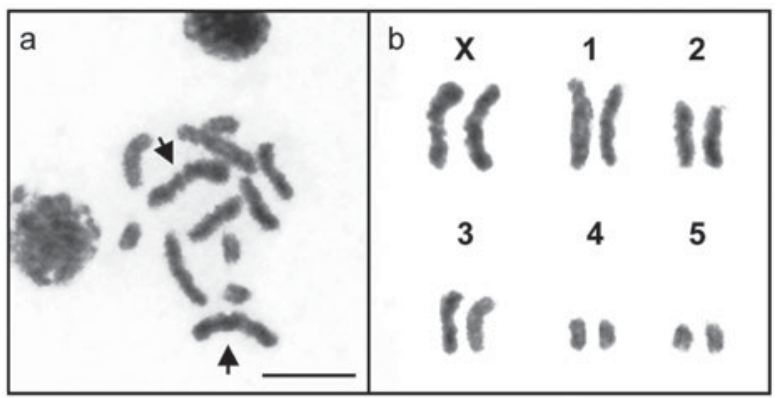

Fig. 2. Metaphase plate of the M. persicae strain Ferrara 03 stained with (a) Giemsa and (b) relative karyotype. Arrows indicate $X$ chromosomes. Bar corresponds to $10 \mu \mathrm{m}$.

were then transferred to minitubes and centrifuged at $350 \mathrm{~g}$ for $3 \mathrm{~min}$. Methanol-acetic acid 3:1 was added to the pellet, which was made to flow up and down for $1 \mathrm{~min}$ through a needle of a $1 \mathrm{ml}$ hypodermic syringe to obtain disgregation of the material followed by a further centrifugation at $1000 \times \mathrm{g}$ for $3 \mathrm{~min}$. This step was repeated with fresh fixative. Finally, the pellet was resuspended in new fixative, and $20 \mu \mathrm{l}$ of cellular suspension was dropped onto clean slides and stained with 5\% Giemsa solution in Soerensen buffer, $\mathrm{pH} 6.8$ for $10 \mathrm{~min}$. Silver staining of nucleolar organizing regions (NORs) was achieved following Howell \& Black (1980). Slides were examined using a Nikon Eclipse 80i fluorescence microscope with UV filters, and photographs were taken using Nikon digital sight DS-U1. Morphometric analyses of mitotic plates were carried out on 30 metaphases using the software MicroMeasure, freely available at the Biology Department at Colorado State University website (http://rydberg.biology.colostate.edu/ MicroMeasure). Male induction for Salerno 03, Pescara 02, Cosenza 02 and Pisa 01 strains was evaluated by exposing parthenogenetic female aphids to short photoperiods $(8 \mathrm{~h}$ light:16h dark) according to Crema (1979).

\section{Results}

The analysis of mitotic cells of embryos, obtained from parthenogenetic females, confirmed that $2 n=12$ is the standard chromosome number in M. persicae (fig. 2), but 14 out of 66 strains analysed showed intraspecific karyotype variants due to both structural and numerical variations in chromosome complements (table 1, figs 3-6).

The most frequent chromosomal rearrangement found in Italian populations is related to the A1-3 reciprocal translocation, which was found either alone (fig. 3) or together with an A3 fission (in one strain; fig. 6a, b). Other chromosome fissions involved A3 (found in two cases; fig. 4) and A4 (found in three cases; fig. 5), whereas a strain possessing 14 chromosomes as a consequence of both $\mathrm{X}$ and $\mathrm{A} 3$ fissions was also found (fig. 6c, d). Lastly, we identified a strain showing an intra-individual chromosome mosaicism due to the presence of mitotic plates with 12 (24\% of the observed plates) and $13(76 \%)$ chromosomes as a consequence of an A3 fission (fig. 4b).

NOR staining (figs $3 a, c, g$, h and $6 c$ ) revealed the presence of heteromorphism in the size of rDNA genes in strains Salerno 3 (fig. 4c) and Cosenza 2 (fig. 6c) and evidenced that the fission of the $X$ chromosomes observed in Cosenza 2 always occurred in the $X$ chromosome bearing the smallest
NOR-positive telomere and involved the $\mathrm{X}$ telomere opposite to the rDNA-bearing one (fig. 6c).

Considering the geographical distribution, it is evident that almost all karyotype variations (11 out of 14) were present in central and southern Italian regions, whereas only three were found in northern locations. Furthermore, all but one of the strains collected on tobacco showed chromosomal rearrangements; and, in particular, all the strains possessing the A1-3 reciprocal translocation were found on this plant and were red in colour.

Male induction revealed that the M. persicae strains Salerno 03, Pescara 02 and Cosenza 02, all possessing different kinds of karyotype variations, are anholocyclic since it was not possible to induce the sexual generation differently from that obtained under the same experimental conditions with the $M$. persicae strain Pisa 1, which showed a normal karyotype.

\section{Discussion}

The typical aphid karyotype consists of pairs of rod-like chromosomes, whose number is typically stable within a genus, as shown in the large genus Aphis, where the typical chromosome number is eight with the exception of $A$. farinosa with $2 n=6$ (Blackman, 1980; Hales et al., 1997). Nevertheless, exceptions have been published as revealed in the genus Amphorophora, where the chromosome number varies from $2 n=4$ to $2 n=72$ (Blackman, 1980).

Rearrangements most commonly involved autosomes, as shown in $M$. persicae, where, despite a standard chromosome number of $2 n=12$, several strains possessing karyotypes consisting of 11-14 chromosomes have previously been reported (Blackman, 1980). On the contrary, Hales (1989) and Monti et al. (2012) demonstrated a complex pattern of associations and fissions occurring on both autosomes and $\mathrm{X}$ chromosomes in Schoutedenia lutea (van der Goot) (Hemiptera: Aphididae) and M. persicae, respectively, suggesting different scenarios for understanding aphid karyotype evolution.

The most common chromosomal variant described in M. persicae complement is a reciprocal translocation between the first and the third autosome pairs, leading to females with $2 n=12$ karyotype showing a marked structural heterozygosity (Blackman, 1980).

The empirical data, as presented in this paper, reveal for the first time that this chromosomal aberration also occurs in Italy since seven strains showed karyotype variations due to the A1-3 reciprocal translocation. In view of the absence of any primary constriction, which is typical of the holocentric chromosomes, together with the lack of specific banding patterns after conventional banding procedures, we combined procedures of standard chromosome staining (such as Giemsa and silver staining) with chromosome length evaluation. In particular, we used silver staining to confirm the exclusive localization of NORs regions on $\mathrm{X}$ chromosome telomeres in $M$. persicae and analyzed the involvement of sex chromosomes in the translocation event (Manicardi et al., 2002). Afterwards, in the absence of any other cytogenetic markers, the morphometric analysis was employed to identify autosomes A1 and A3 as the chromosomes engaged in the rearrangement.

According to the literature, a link exists between the A1-3 chromosomal reciprocal translocation and resistance to organophosphate and carbamate insecticides due to E4 gene amplification (Blackman et al., 1995), perhaps involving the removal of a repressor gene away from the structural genes in 


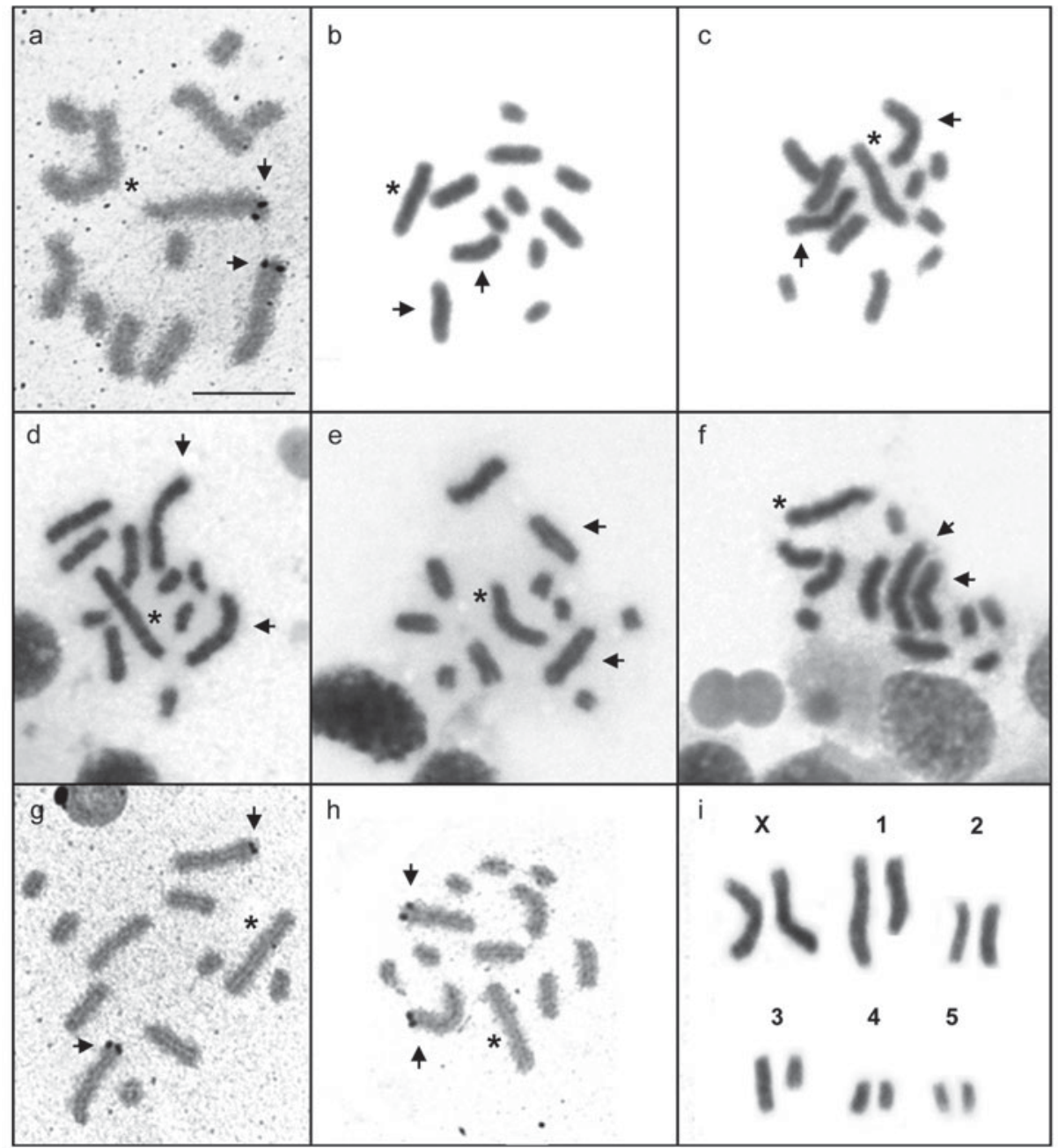

Fig. 3. M. persicae chromosome complements showing A1-3 reciprocal translocation. (a) Benevento 01 is silver stained, (b) Salerno 01, (c) Chieti 02 and (e) Chieti 03 are stained with Giemsa, whereas (d, g) Chieti 1 and (f, h) Chieti 4 are both Giemsa and silver stained. The (i) karyotype is derived from (c) Chieti 02. Arrows indicate X chromosomes. Asterisks indicate A1-3 translocated chromosomes. Bar corresponds to $10 \mu \mathrm{m}$.

controls (Blackman et al., 1978). Preliminary data involving PCR and Southern blot analysis revealed that, in one of the Italian populations with this chromosomal aberration (Chieti 1), the FE4 gene (electrophoretically fast variant (allele) of the normal expressed carboxylesterase 4 (E4) enzyme) only was present (Rivi et al., 2009). This strain showed a moderate increase in esterase activity and was considered an S/R1 (susceptible/first resistance level) strain sensu Devonshire et al. (1992). The aforementioned data allows us to suggest that this is the first $M$. persicae strain possessing the A1-3 chromosomal reciprocal translocation linked to an FE4 and not directly related to a high level of esterase-based insecticide resistance. Experiments currently in progress are aimed to extend this experimental procedure to all Italian strains possessing A1-3 reciprocal translocations, in order to better clarify the relationships between this chromosomal rearrangement and the insecticide resistance in $M$. persicae populations.

Other fissions relatively frequent in the studied Italian M. persicae populations occurred at autosomes 3 and 4, whereas in one case only the fission involved the $X$ chromosome. Different autosome fragmentations have been repeatedly described in $M$. persicae populations collected worldwide, whereas the $X$ fragmentation has been observed only in a $M$. persicae laboratory strain characterised by an extensive chromosomal mosaicism (Monti et al., 2012). In this connection, it must be emphasized that in both such cases, the $X$ fission occurs in $X$ chromosomes possessing a low number of rDNA genes and in the telomeric region opposite to the NORsbearing one. The recurrent fission of the same chromosomes in the same region argues that the $M$. persicae genome possesses some fragile/labile sites that could be the basis for the observed changes in the chromosome number.

For many years, chromosome evolution has been generally explained by considering the random-breakage model (Becker \& Lenhard, 2007). On the contrary, a number of comparative cytogenetic studies evidences a relationship between chromosomal rearrangements and specific chromosomal architecture and suggests a role of the repetitive DNAs in chromosome 


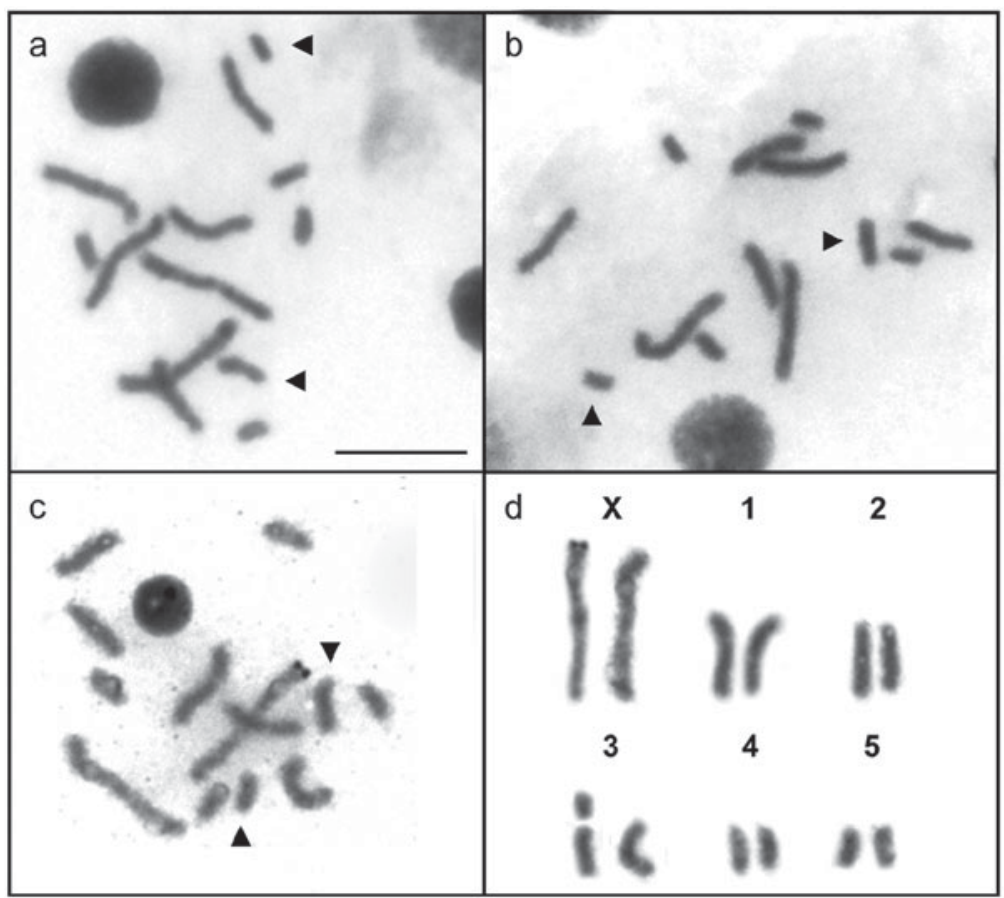

Fig. 4. M. persicae chromosome complements showing A3 fission. (a) Forlì 01 and (b) Salerno 02 are stained with Giemsa, whereas (c) Salerno 03 and (d) its relative karyotype are silver stained. Arrow heads indicate chromosomes involved in the fission. Bar corresponds to $10 \mu \mathrm{m}$.

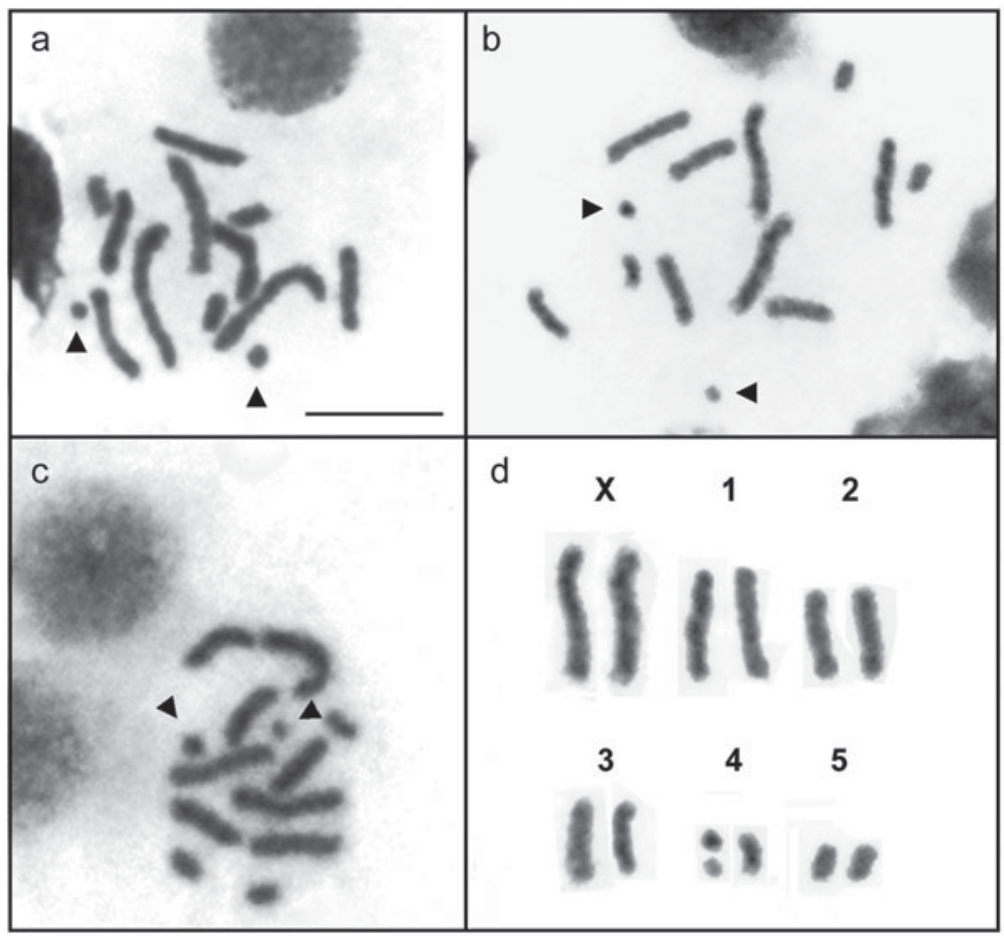

Fig. 5. Giemsa staining of M. persicae chromosome complements showing A4 fission: (a) Cosenza 01, (b) Ravenna 06 and (c) Piacenza 10. The (d) karyotype is derived from (b) Ravenna 06. Arrow heads indicate chromosomes involved in the fission. Bar corresponds to $10 \mu \mathrm{m}$.

rearrangements. The nature of the repetitive DNA within chromosomal breakpoint regions varies significantly, from clusters of rRNA and tRNA genes to simple di- and tri-nucleotide expansions (Caceres et al., 1999; Carlton et al., 2002; Coghlan \& Wolfe, 2002; Kellis et al., 2003; Renciuk et al., 2011). The data reported in this paper confirmed recent 


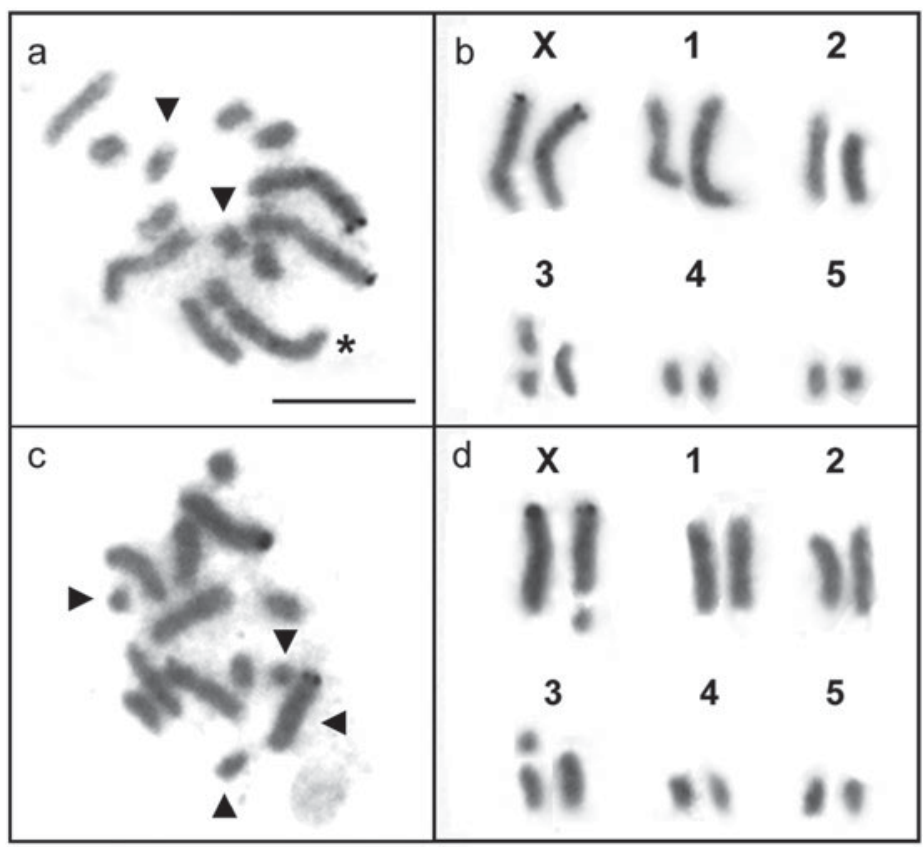

Fig. 6. (a) Pescara 02 complement stained with $\mathrm{AgNO}_{3}$ and (b) relative karyotype. (c) Cosenza 02 complement silver stained with (d) relative karyotype. Arrow heads indicate chromosomes involved in the fissions. Asterisk indicates A1-3 translocated chromosomes. Bar corresponds to $10 \mu \mathrm{m}$.

observations regarding the recurrent fission of the same chromosomes in the same region (Monti et al., 2012), allowing us to further support the hypothesis concerning the presence of fragile/labile sites in the M. persicae holocentric chromosomes.

Chromosomal rearrangements in aphids have been hypothesized to affect some complex phenotypic traits, such as the host plant choice (Blackman, 1987; ffrench-Constant et al., 1988). For example, karyotypic variants observed in the corn leaf aphid Rhopalosiphum maidis (Fitch) have been associated with changes in the host choice. Similarly, an association of chromosome number with host plant has been described within the Sitobion genus, which shows $2 n=12$ on ferns and $2 n=18$ on grasses (Brown \& Blackman, 1988; Hales et al., 1997).

A peculiar example of host adaptation concerns $M$. persicae strains feeding on tobacco. Morphometric analyses of specific taxonomic markers revealed that they are distinguishable from those living on other host plant so that the tobaccofeeding form was elevated to the status of a separate species by Blackman (1987). Further molecular evidences failed to confirm the genetic isolation of the population living on tobacco (Field et al., 1994; Clements et al., 2000), although other data, as well as behavioural/pheromonal evidence, suggest that the two forms undergone some significant degree of ecological-evolutionary divergence (Kephalogianni et al., 2002; Margaritopolous et al., 2003; Blackman et al., 2007).

Our data put in evidence that all but one of the strains collected on tobacco plants showed karyotype variations, whereas only four of the 56 population collected on other hosts (corresponding to about 7\% of the total) displayed chromosomal rearrangements. A suggestive explanation for the observed relationships between chromosomal rearrangements and tobacco plants could rely in the clastogenic effect of nicotine.

Nicotine is a naturally occurring alkaloid found primarily in members of the solanaceous plant family, including Nicotiana tabacum. Several reports showed that nicotine, as a consequence of DNA replication fork stress (Richards, 2001; Freudenreich, 2005), produces genotoxic effects on Chinese hamster ovarian (CHO) cells (Trivedi et al., 1990, 1993) and sister chromatid exchanges and chromosome aberrations in bone marrow cells of mice (Sen et al., 1991). Extensive chromosomal rearrangements have also been described in a mice population known as 'tobacco mice' since they live close to kiln for drying tobacco (Fraguedakis-Tsolis et al., 1997). In addition, DNA fragmentation by nicotine has been demonstrated both in peripheral lymphocytes (Sassen et al., 2005) and in human spermatozoa (Arabi, 2004). Nicotine, together with ultraviolet exposure, has also been considered an exogenous factor which can contribute to the generation of mutations which could be at the basis of chromosomal mosaicism (De, 2011), a very rare phenomenon we have observed in Salerno 02 , one of the strains collected on tobacco plants.

Even if there are no literature data analyzing nicotine effects on organisms possessing holocentric chromosomes, the previously reported data allow us to propose at least that chromosome architecture, rather than random breakages, has a pivotal role in aphid chromosome evolution and rearrangements.

The high telomerase expression, previously reported in M. persicae (Monti et al., 2011), that stabilized chromosomes involved in fragmentations, coupled to reproduction by obligate apomictic parthenogenesis, could be at the basis of the stabilization of the observed chromosome instability on M. persicae strains collected on tobacco plants favouring the inheritance of the variant karyotypes. 


\section{References}

Arabi, M. (2004) Nicotinic infertility: assessing DNA and plasma membrane integrity of human spermatozoa. Andrologia 36, 305-310.

Becker, T.S. \& Lenhard, B. (2007) The random versus fragile breakage models of chromosome evolution: a matter of resolution. Molecular Genetics and Genomics 278, 487-491.

Blackman, R.L. (1971) Variation in the photoperiodic response within natural populations of Myzus persicae (Sulz.). Bulletin of Entomological Research 60, 533-546.

Blackman, R.L. (1980) Chromosome numbers in the Aphididae and their taxonomic significance. Systematic Entomology 5, $7-25$.

Blackman, R.L. (1987) Morphological discrimination of a tobaccofeeding form from Myzus persicae (Sulzer) (Hemiptera: Aphididae), and a key to new world Myzus (Nectarosiphon) species. Bulletin of Entomological Research 77, 713-730.

Blackman, R.L., Takada, H. \& Kawakami, K. (1978) Chromosomal rearrangement involved in insecticide resistance of Myzus persicae. Nature 271, 450-452.

Blackman, R.L., Spence, J.M., Field, L.M. \& Devonshire, A.L. (1995) Chromosomal location of the amplified esterase genes conferring resistance to insecticides in Myzus persicae (Homoptera: Aphididae). Heredity 75, 297-302.

Blackman, R.L., Malarky, G. \& Margaritopoulos, J.T. (2007) Distribution of common genotypes of Myzus persicae (Hemiptera: Aphididae) in Greece, in relation to life cycle and host plant. Bulletin of Entomological Research 97, 253-263.

Brown, P.A. \& Blackman, R.L. (1988) Karyotype variation in the corn leaf aphid, Rhopalosiphum maidis (Fitch), species complex (Hemiptera: Aphididae) in relation to host-plant and morphology. Bulletin of Entomological Research 78, 351-363.

Caceres, M., Ranz, J.M., Barbadilla, A., Long, M. \& Ruiz, A. (1999) Generation of a widespread Drosophila inversion by a transposable element. Science 285, 415-418.

Carlton, J.M., Angiuoli, S.V., Suh, B.B., Kooij, T.W., Pertea, M., Silva, J.C., Ermolaeva, M.D., Allen, J.E., Selengut, J.D., Koo, H.L., Peterson, J.D., Pop, M., Kosack, D.S., Shumway, M.F., Bidwell, S.L., Shallom, S.J., van Aken, S. E., Riedmuller, S.B., Feldblyum, T.V., Cho, J.K., Quackenbush, J., Sedegah, M., Shoaibi, A., Cummings, L. M., Florens, L., Yates, J.R., Raine, J.D., Sinden, R.E., Harris, M.A., Cunningham, D.A., Preiser, P.R., Bergman, L. W., Vaidya, A.B., van Lin, L.H., Janse, C.J., Waters, A.P., Smith, H.O., White, O.R., Salzberg, S.L., Venter, J.C., Fraser, C.M., Hoffman, S.L., Gardner, M.J. \& Carucci, D.J. (2002) Genome sequence and comparative analysis of the model rodent malaria parasite Plasmodium yoelii yoelii. Nature 419, 512-519.

Clements, K.M., Sorenson, C.E., Wiegmann, B.M., Neese, P.A. \& Roe, R.M. (2000) Genetic, biochemical, and behavioural uniformity among populations of Myzus nicotianae and Myzus persicae. Entomologia Experimentalis et Applicata 95, 269-281.

Coghlan, A. \& Wolfe, K.H. (2002) Fourfold faster rate of genome rearrangement in nematodes than in Drosophila. Genome Research 12, 857-867.

Crema, R. (1979) Egg viability and sex determination in Megoura viciae (Homoptera: Aphididae). Entomologia Experimentalis et Applicata 26, 152-156.

De, S. (2011) Somatic mosaicism in healthy human tissues. Trends in Genetics 27, 217-223.
Devonshire, A.L., Devine, G.J. \& Moores, G.D. (1992) Comparison of microplate esterase assays and immunoassay for identifying insecticide resistant variants of Myzus persicae (Homoptera: Aphididae). Bulletin of Entomological Research 82, 459-463.

ffrench-Constant, R.H., Byrne, F.J., Stribley, M.F. \& Devonshire, A.L. (1988) Rapid identification of the recently recognised Myzus antirrhinii (Macchiati) (Hemiptera: Aphididae) by polyacrylamide gel electrophoresis. Entomologist 107, 20-23.

Field, L.M., Javed, N., Stribley, M.F. \& Devonshire, A.L. (1994) The peach-potato aphid Myzus persicae and the tobacco aphid Myzus nicotianae have the same esterase-based mechanisms of insecticide resistance. Insect Molecular Biology 3, 143-148.

Fraguedakis-Tsolis, S., Hauffe, H.C. \& Searle, J.B. (1997) Genetic distinctiveness of a village population of house mice: Relevance to speciation and chromosomal evolution. Proceedings of the Royal Society of London, Series B: Biological Science 264, 355-360.

Freudenreich, C.H. (2005) Molecular mechanisms of chromosome fragility. ChemTracks-Biochemistry and Molecular Biology 18, 141-152.

Hales, D.F. (1989) The chromosomes of Schoutedenia lutea (Homoptera, Aphidoidea, Greenideinae), with an account of meiosis in the male. Chromosoma 98, 295-300.

Hales, D.F., Tomiuk, J., Wohrmann, K. \& Sunnucks, P. (1997) Evolutionary and genetic aspects of aphid biology: A review. European Journal of Entomology 94, 1-55.

Howell, W.M. \& Black, D.A. (1980) Controlled silver-staining of nucleolus organizer regions with a protective colloidal developer: a 1-step method. Experientia 36, 1014-1015.

Kellis, M., Patterson, N., Endrizzi, M., Birren, B. \& Lander, E.S. (2003) Sequencing and comparison of yeast species to identify genes and regulatory elements. Nature 423, 241-254.

Lauritzen, M. (1982) Q-Band and G-Band Identification of 2 chromosomal rearrangements in peach-potato aphids, Myzus persicae (Sulzer), resistant to insecticides. Hereditas 97, 95-102.

Manicardi, G.C., Mandrioli, M., Bizzaro, D. \& Bianchi, U. (2002) Cytogenetic and molecular analysis of heterochromatic areas in the holocentric chromosomes of different aphid species. pp. 47-56 in Sobti, R.C., Obe, G. \& Athwal, R.S. (Eds), Some Aspects of Chromosome Structure and Functions. New Delhi, India, Narosa Publishing House.

Kephalogianni, T.E., Tsitsipis, J.A. \& Margaritopoulos, J.T. (2002) Variation in the life cycle and morphology of the tobacco host-race of Myzus persicae (Hemiptera: Aphididae) in relation to its geographical distribution. Bulletin of Entomological Research 92, 301-307.

Loxdale, H.D. \& Lushai, G. (2003) Rapid changes in clonal lines: the death of a 'sacred cow'. Biological Journal of the Linnean Society 79, 3-16.

Margaritopoulos, J.T., Blackman, R.L. \& Tsitsipis, J.A. (2003) Coexistence of different host-adapted forms of the Myzus persicae group (Hemiptera: Aphididae) in southern Italy. Bulletin of Entomological Research 93, 131-135.

Monti, V., Giusti, M., Bizzaro, D., Manicardi, G.C. \& Mandrioli, M. (2011) Presence of a functional (TTAGG) telomere-telomerase system in aphids. Chromosome Research 19, 625-633.

Monti, V., Mandrioli, M., Rivi, M. \& Manicardi, G.C. (2012) The vanishing clone: karyotypic evidence for extensive intraclonal genetic variation in the peach potato aphid, Myzus persicae (Hemiptera: Aphididae). Biological Journal of the Linnean Society 105, 350-358. 
Renciuk, D., Kypr, J. \& Vorlickova, M. (2011) CGG Repeats associated with fragile $\mathrm{X}$ chromosome form left-handed Z-DNA structure. Biopolymers 95, 174-181.

Richards, R. (2001) Fragile and unstable chromosomes in cancer: causes and consequences. Trends in Genetics 17, 339-345.

Rivi, M., Mazzoni, E., Criniti, A., Cassanelli, S., Bizzaro, D. \& Manicardi, G.C. (2009) Relationship between chromosomal translocation and FE4 gene amplification in an Italian population of the peach-potato aphid Myzus persicae (Hemiptera: Aphididae). Redia 92, 229-231.

Sassen, A., Richter, E., Semmler, M., Harreus, U., Gamarra, F. \& Kleinsasser, N. (2005) Genotoxicity of nicotine in mini-organ cultures of human upper aerodigestive tract epithelia RID A-3601-2008. Toxicological Sciences 88, 134-141.

Sen, S., Sharma, A. \& Talukder, G. (1991) Inhibition of clastogenic effects of nicotine by chlorophyllin in mice bone-marrow cells in vivo. Phytotherapy Research 5, 130-133.

Spence, J.M. \& Blackman, R.L. (1998) Chromosomal rearrangements in the Myzus persicae group and their evolutionary significance. pp. 113-118 in Nieto Nafría, J.M. \& Dixon, A.F. G. (Eds) Chromosomal Rearrangements in the Myzus persicae
Group and their Evolutionary Significance. León, Spain, Universidad De León Secretariado de Publicacions.

Spence, J.M. \& Blackman, R.L. (2000) Inheritance and meiotic behaviour of a de novo chromosome fusion in the aphid Myzus persicae (Sulzer). Chromosoma 109, 490-497.

Trivedi, A.H., Dave, B.J. \& Adhvaryu, S.G. (1990) Assessment of genotoxicity of nicotine employing in vitro mammalian test system. Cancer Letters 54, 89-94.

Trivedi, A.H., Dave, B.J. \& Adhvaryu, S.G. (1993) Genotoxic effects of tobacco extract on Chinese hamster ovary cells. Cancer Letters 70, 107-112.

White, M.J.D. (1973) Animal Cytology and Evolution. Cambridge, UK, Cambridge University Press.

Wrensch, D.L., Ketheley, J.B. \& Norton, R.A. (1994) Cytogenetic of holokinetic chromosomes and inverted meiosis: keys to the evolutionary success of mites with generalization on eukaryotes. pp. 282-343 in Houck, M.A. (Ed.), Mites: Ecological and Evolutionary Analysis of Life-History Patterns. New York, USA, Chapman \& Hall.

Yang, X.W. \& Zhang, X. (2000) Karyotype polymorphism in different geographic populations of green peach aphid Myzus persicae (Sulzer) in China. Entomologia Sinica 7, 29-35. 